\title{
Diferenciais de mortalidade em um hospital filantrópico: a Santa Casa de Misericórdia de Campinas (1876-1885)
}

\author{
Matheus Alves Albino* \\ Renato Leite Marcondes ${ }^{\star \star}$
}

\begin{abstract}
A partir da documentação das Matrículas de Enfermos e Relatórios dos Provedores da Santa Casa de Misericórdia de Campinas, traçamos o perfil dos atendidos e analisamos os diferenciais de mortalidade entre escravos e livres, brasileiros e estrangeiros nos primeiros anos de funcionamento desse hospital filantrópico. Os enfermos eram predominantemente homens em idade ativa, fortemente relacionados com a mão de obra disponível para comportar a expansão agrícola e dos serviços urbanos. Por meio de uma técnica de análise do diferencial de mortalidade baseada na decomposição de Oaxaca-Blinder, concluímos que, apesar de as características de escravos favorecerem um diferencial de mortalidade reduzido e de haver incentivos econômicos em seu tratamento, a mortalidade escrava foi superior possivelmente devido às condições de vida impostas pelo cativeiro. Entre a população livre, brasileiros apresentaram mortalidade superior à de estrangeiros. As hipóteses levantadas foram a procura tardia de auxílio por parte dos nacionais, diferenças na estrutura etária e a forte característica de sub-representação feminina na população estrangeira.
\end{abstract}

Palavras-chave: Mortalidade hospitalar. Diferenciais de mortalidade. Escravos. Santa Casa de Misericórdia.

\footnotetext{
* Instituto de Filosofia e Ciências Humanas da Universidade Estadual de Campinas (Unicamp), Campinas-SP, Brasil (albino@ usp.br; https://orcid.org/0000-0003-3102-7663).

** Departamento de Economia, Faculdade de Economia, Administração e Contabilidade de Ribeirão Preto (FEA-RP) da Universidade de São Paulo, Ribeirão Preto-SP, Brasil (rlmarcon@usp.br; https://orcid.org/0000-0002-7002-621X).
} 


\section{Introdução}

A segunda metade do século XIX foi caracterizada por grandes mudanças na composição da população, decorrentes do crescimento da população livre por meio de reprodução natural, da emancipação dos escravos e da imigração (EISENBERG, 1989, p. 224). Nesse cenário, a cidade de Campinas, localizada no chamado oeste paulista, despontou como uma das maiores e mais importantes produtoras de café de São Paulo. 0 município experimentou desenvolvimento econômico, transformações no mundo do trabalho, crescimento populacional e ampliação do quadro urbano, mas também foi atingido pelos surtos das doenças infectocontagiosas que impactaram dramaticamente a vida no mundo urbano e rural, sua economia e demografia. Desde a lepra e a varíola no início e metade do século XIX até a febre amarela em suas décadas finais, as doenças tiveram importante lugar no rol de preocupações da população e do poder público.

Esse período de grande impacto das doenças infectocontagiosas e mortalidade elevada é comumente caracterizado pelos demógrafos como uma fase pré-transicional, referindo-se à teoria da transição epidemiológica de Omran (1971), desenvolvida para explicar os avanços na saúde em países industrializados desde o século XVIII. De acordo com a teoria, alguns condicionantes biológicos, socioeconômicos, políticos e culturais que afetam os padrões de vida, hábitos de saúde, higiene e nutrição favoreceram uma mudança da influência de doenças infectocontagiosas para doenças cardiovasculares.

Numa tentativa de colocar em perspectiva histórica a transição de mortalidade e epidemiológica, Horiuchi (1999) postula que, a partir do momento em que são controladas as doenças infectocontagiosas, ocorre a redução na mortalidade perinatal e materna, por essas doenças e por problemas nutricionais. A partir desse momento as moléstias mais expressivas passam a ser as cardiovasculares e degenerativas. 0 autor afirma que países em desenvolvimento, como o Brasil, iniciaram uma transição tardia em relação a países da Europa ocidental.

De maneira geral, a literatura brasileira é relativamente convergente em torno da afirmativa de que as condições de vida na segunda metade do século XIX eram insalubres e a mortalidade, em especial a infantil e a escrava,era extremamente elevada. ${ }^{1}$ Esse panorama somente foi alterado ao longo da primeira metade do século XX, com as medidas sanitárias e descobertas da medicina sobre as doenças infectocontagiosas. Desde a década de 1970, ao discutirem a evolução dos serviços de saúde no Brasil, Singer, Campos e Oliveira (1978) associavam nosso período de análise à predominância das doenças infectocontagiosas com desorganização dos serviços de saúde e prática médica baseada em grande parte por conhecimentos tradicionais.

Um dos símbolos do período das grandes epidemias foram os hospitais de isolamento. Em Campinas, o Asilo de Morféticos (1863) confinava de maneira compulsória os portadores

\footnotetext{
${ }^{1}$ Cabe destacar a obra pioneira de Iraci del Nero da Costa (1979) sobre a mortalidade em Vila Rica (MG) no século XVIII e início do XIX baseada em registros paroquiais. Desde então, vários estudos abordaram a mortalidade por meio dessa fonte.
} 
da doença assim que fossem identificados pelo fiscal da Câmara. Da mesma forma, para conter os casos de varíola e outras enfermidades que atingiram várias localidades da província, foi construído em 1874 o Hospital de Variolosos.Dadas as condições de vida precárias, a população pobre se tornou o alvo das doenças. Uma massa de excluídos pela pobreza ou pelas doenças morreu no desamparo ou confinada em um dos ambientes de isolamento da cidade (LAPA, 1996, p. 217).

Essa população trouxe a necessidade da organização de um sistema de assistência, colocado em prática pelos hospitais filantrópicos. Nesse contexto, as fundações de estabelecimentos como a Santa Casa de Misericórdia de Campinas em 1876 e do hospital da Sociedade Portuguesa de Beneficência (1879) significaram importantes marcos para a prática assistencial da cidade e representaram um processo de institucionalização da filantropia, que até então era pulverizada em ações assistenciais e em irmandades e confrarias (ROCHA, 2005, p. 105).

Embora as conexões entre escravidão e condições de saúde da população tenham sido desenvolvidas por uma bibliografia relativamente sólida, sabe-se muito pouco sobre a assistência à população escravizada e livre pobre oferecida por instituições filantrópicas como as Misericórdias. Além disso, o uso de estatísticas hospitalares para reconstituir elementos da morbimortalidade das populações do passado ainda é raro na produção da demografia histórica brasileira. Nesse sentido, destaca-se um maior esforço da literatura internacional em utilizar esse tipo de fonte com um olhar demográfico. Steven Cherry (1980) verificou, por meio dos registros hospitalares de províncias inglesas ao longo dos séculos XVIII e XIX, que no geral as condições sanitárias favoreciam uma redução da mortalidade, muito embora essa situação tenha se deteriorado ao longo do século XIX com o crescimento populacional e maior demanda por recursos do que os hospitais poderiam oferecer.

Para as principais cidades do Brasil, autores como Laima Mesgravis (1977) para São Paulo, Russell-Wood (1981) para a Bahia e Mary Karasch (2000) para o Rio de Janeiro trabalharam especificamente com registros das Santas Casas. Com o avanço das investigações, surgiram estudos para outras cidades, como o de Leila Alves Rocha (2005) para Campinas, Nikelen Witter (2007) para Porto Alegre, Claudia Tomaschewski (2007) para a Santa Casa de Pelotas, entre outros. Nesses trabalhos, nota-se que o perfil dos atendidos era majoritariamente masculino e em idade ativa, sendo que a mortalidade foi bastante elevada, em torno de $15 \%$ das entradas. ${ }^{2}$ Isso levou a serem entendidos pela população como antecâmaras da morte, lugares onde se ia para morrer (TELAROLLI JÚNIOR, 2014, p. 10). Os primeiros Relatórios da Provedoria da Santa Casa de Campinas confirmam essa tendência (RELATÓRIO DOS PROVEDORES, 1877, p. 3-4).

A Misericórdia de Campinas atendeu desde livres nacionais e estrangeiros até cativos entre 1876 e 1885 . Não obstante o pagamento ou não das custas pelos pacientes, não

\footnotetext{
2 Segundo os Relatórios dos presidentes de Província, isso pode ser confirmado para as Santas Casas da Bahia (BAHIA, 1877), Porto Alegre (PORTO ALEGRE, 1878), Rio de Janeiro (BRASIL, 1878), Campinas e Santos (SÃO PAULO, 1877), pelo menos para o início do período em análise.
} 
observamos relatos de discriminação no tratamento, de forma que brasileiros, estrangeiros e escravos pareciam ter condições semelhantes de cuidado ao entrarem no hospital, como relata o provedor:

Nem a condição do escravo sofre dentro destas paredes distinção de tratamento, achando tudo comum com os demais que lá fora se extremam por livres. Como na sagrada mesa, comungam todos igualmente aqui nos benefícios da caridade (RELATÓRIO DOS PROVEDORES, 1883, p. 11).

O Relatório dos Provedores (1877, p. 44) traz indícios de que o estrangeiro, apesar de enfrentar dificuldades na obtenção de documentos necessários para admissão, procurou os cuidados do hospital em estados menos graves, dirigindo-se ao hospital assim que se pronunciasse a moléstia. Os nacionais, por outro lado, procuraram a Santa Casa só muito tarde e em lastimáveis condições de saúde.

0 estrangeiro conhece o hospital e sabe-lhe o valor; encontrou-o sempre em todos os seus sofrimentos físicos - colhendo-lhe os benefícios que lhe podia dar; não pergunta a ninguém se lá se morre, porque sabe que mais riscos correrá abandonado, vítima ao mesmo tempo da moléstia e da miséria, e ao sentir-se doente procura-0, como abrigo de pobreza e socorro de ciência (RELATÓRIO DOS PROVEDORES, 1877, p. 44).

No entanto, ainda está para ser feita uma exploração mais aprofundada dos registros hospitalares no que diz respeito aos enfermos e à mortalidade diferencial entre escravos e livres, nacionais e estrangeiros. Neste artigo, examinamos a documentação da Santa Casa de Misericórdia de Campinas - ainda pouco explorada pelos estudiosos - para traçar o perfil dos enfermos e a mortalidade diferencial nesse hospital filantrópico durante a segunda metade do século XIX.

Focalizou-se o período entre 1876 e 1885, correspondente aos nove primeiros anos dos registros. 0 marco inicial significou o ano da fundação do Hospital de Misericórdia de Campinas e, para nosso exercício, foi suficiente encerrar a análise em 1885, visto que compreende os anos de registros mais sistemáticos de escravos nas enfermarias.

\section{Dados}

No passado assim como na atualidade, o acesso à saúde e a relação entre desigualdade socioeconômica e mortalidade emergem como grandes questões da demografia. Particularmente, a literatura sobre o tema concentrou-se em desenvolver e consolidar técnicas de análise das taxas de mortalidade e morbidade para uma determinada população, com o apoio de fontes qualitativas para melhor investigar os níveis, padrões e tendências da mortalidade.

As estatísticas de mortalidade para a segunda metade do século XIX estão longe de um estado desejado. Nesse período, o Brasil passou pela transição entre os registros paroquiais e o registro civil, em consonância com várias outras transformações sociais e políticas. A precariedade desses registros é uma barreira natural, porém não totalmente impeditiva, e induz à busca de outras fontes como as estatísticas hospitalares para complementar o 
entendimento do universo da escravidão. A bibliografia que utiliza como fontes os registros disponíveis em hospitais do século XIX para investigar a mortalidade de escravos e livres, no entanto, ainda é relativamente escassa. Um dos motivos é que, embora as casas de caridade no interior paulista recebessem escravos, muito provavelmente esse não era o lugar mais frequente para o tratamento, dado que algumas fazendas contavam com enfermarias e o tratamento por meio de curandeiros e pessoas leigas era uma prática comum. ${ }^{3}$

A partir dos livros de matrículas de enfermos da Santa Casa de Misericórdia de Campinas, é possível ter uma noção mais acurada do fluxo de pessoas atendidas pelo hospital. As possibilidades de estudo para essa fonte são as mais variadas. As informações disponíveis nos registros são em grande parte completas, trazendo o nome do paciente, idade, estado conjugal, nacionalidade, ocupação, naturalidade, local de residência, data de entrada e saída, destinação final (entre morte, cura e melhora), doenças/causa mortis e um campo de informação de remetente.

O campo de remetente traz informações sobre a classe do enfermo: os pobres eram atendidos gratuitamente pela caridade, enquanto os pensionistas pagavam uma diária para tratamento na Santa Casa. Além disso, o hospital atendia soldados. Os escravos tinham suas diárias pagas pelos seus proprietários. A partir dessa informação, é possível fazer o cruzamento nominativo dos principais escravistas que remetiam enfermos para o Hospital. Geralmente, escravos não possuíam identificação de sobrenome, mas a adoção desse método de identificação provoca graves distorções. ${ }^{4}$ Dessa forma, identificamos escravos por meio da anotação explícita de sua condição ou da informação do remetente, o que nos fornece um número de escravos compatível com o dos quadros gerais dos relatórios de administração da Santa Casa, elaborados bianualmente pelo provedor da Irmandade de Misericórdia.

Os livros também contêm observações sobre as moléstias dos enfermos. Essa informação dá sabor à leitura da documentação, mas é repleta de termos que dificultam uma análise cuidadosa da morbidade associada a determinadas doenças sem que se estabeleça uma classificação geral das moléstias e das expressões diagnósticas, além de provavelmente estarem sujeitas a erros de diagnóstico, motivo pelo qual aqui reduzimos a interpretação da mortalidade hospitalar a termos de mortalidade geral.

\section{Métodos}

Uma das contribuições da demografia histórica para a análise da mortalidade foi o estudo e o desenvolvimento de métodos de identificação de períodos de crise, como o Método de Dupâquier. Em outra frente, a análise dos diferenciais de mortalidade nos beneficia com

\footnotetext{
${ }^{3}$ Sobre as práticas de cura que eram utilizadas no século XIX, com o auxílio de parteiras, curandeiros e sangradores, bem como a saúde dos escravos, ver Pôrto (2006).

${ }^{4}$ Ao observar os registros hospitalares do Touro Infirmary Hospital, em New Orleans, Pritchett e Yun (2009, p. 5) assumiram que os enfermos sem sobrenome, cerca de $52 \%$ da amostra, eram escravos. Na Santa Casa de Campinas, os casos de livres pobres que não possuem sobrenome e de escravos com sobrenome de seus senhores são recorrentes, o que prejudica a utilização desse método.
} 
a possibilidade de identificar a que fatores se devem as diferenças de comportamento dessa variável demográfica entre duas populações.

Para controlar os efeitos da estrutura etária na comparação de perfis de mortalidade entre duas populações, foram desenvolvidos métodos de padronização e decomposição de diferenças entre taxas ou proporções, a exemplo da técnica desenvolvida por Kitagawa (1955) e estendida para mais variáveis por Das Gupta (1978). De maneira similar, uma série de técnicas baseadas em regressão permite a análise diferencial, utilizando dados desagregados a partir de manipulações algébricas de diferenças (CANUDAS ROMO, 2003, p. 52).

Com a elaboração do banco de dados, tornou-se possível estimar a probabilidade de morte hospitalar por meio de um modelo probit. Essa estratégia foi empregada por autores como Ferrie (2003), utilizando os Censos de 1850 e 1860 dos Estados Unidos, e Green e Hamilton (2013, p. 153), para a Carolina do Norte na década de 1880 . Nossa análise compreendeu os fatores sintetizados no Quadro 1. A variável dependente assume valor 1 para morte no hospital. É razoável ainda esperar que as variáveis de idade e permanência hospitalar apresentem relações não lineares com mortalidade e que o erro de declaração de idade seja significativo, motivo pelo qual optamos por adicionar dois conjuntos de variáveis categóricas correspondentes a grandes grupos etários (0-19 anos, 20-60 anos e maiores de 60 anos) e que representem os quintis da distribuição da permanência hospitalar.

QUADRO 1

Variáveis do modelo de mortalidade hospitalar da Santa Casa de Misericórdia de Campinas

\begin{tabular}{|ll|}
\hline Variáveis & Descrição \\
\hline Variável dependente & Variável binária igual a 1 se o indivíduo faleceu no hospital \\
\hline Idade & $\begin{array}{l}\text { Conjunto de variáveis binárias para idade do paciente em grandes grupos etários } \\
\text { (0-19 anos, 20-60 anos e maiores de 60 anos) }\end{array}$ \\
\hline Masculino & Variável binária igual a 1 se o indivíduo for homem \\
\hline Casado & Variável binária igual a 1 se o indivíduo for casado \\
\hline Campinas & Variável binária igual a 1 se o local de residência for Campinas \\
\hline Permanência hospitalar & Conjunto de controles para quintis da distribuição de permanência \\
\hline Mês e ano de admissão & Conjuntos de controles para meses e anos \\
\hline
\end{tabular}

Diferenças nas taxas de mortalidade de escravos e livres podem ocorrer devido a uma série de características específicas dessas populações ou de doenças que castigaram um grupo mais do que outro. Um dos poucos resultados que apontam para um diferencial de mortalidade favorável ao escravo para estudos do século XIX é apresentado por Pritchett e Yun (2009). Os autores empregaram um método de análise derivado da decomposição de Oaxaca-Blinder, utilizada exaustivamente na Economia do Trabalho para análise do diferencial de salários entre homens e mulheres. Essa decomposição exige que existam dois grupos excludentes entre si (OAXACA, 1973; BLINDER, 1973).

A motivação principal do método é responder perguntas como: quanto das diferenças entre os grupos é atribuído às diferenças nas características ("efeito composição") e 
quanto pode ser atribuído a um efeito residual não explicado, associado às características? É relativamente fácil calcular essa decomposição para regressões lineares. No entanto, o cálculo não é trivial quando utilizamos modelos como o probit (YUN, 2004, p. 275). Em nosso caso, $Y$ é uma variável que assume valor 1 para indivíduos que morreram no hospital e valor 0 , caso contrário. As probabilidades de morte para cada $i$ enfermo da Santa Casa são dadas pela expressão:

$\operatorname{Pr}\left(Y_{i}=1 \mid X\right)=\Phi\left(X_{i} \beta\right)$

Em que $X_{i}$ é o vetor de variáveis explicativas, $\beta$ é o vetor dos coeficientes da regressão e $\Phi(\cdot)$ é a função de distribuição acumulada normal padrão. Seguindo os procedimentos adotados por Pritchett e Yun (2009, p. 248), quando a amostra é suficientemente grande, podemos aproximar uma taxa média de mortalidade geral pela proporção de óbitos observada. Matematicamente,

$\bar{M}=\overline{\Phi(X \beta)}=\frac{1}{N} \sum_{i=1}^{N} \Phi\left(X_{i} \beta\right)$

Com base nesse resultado, estimamos as equações de mortalidade para escravos e livres separadamente, obtendo $\bar{M}_{E}$ e $\bar{M}_{L}$ respectivamente. Calcula-se, a partir disso, uma mortalidade contrafactual de livres caso tivessem os coeficientes dos escravos, isto é, $\tilde{M}_{L}=\overline{\Phi\left(X_{L} \beta_{E}\right)}$. Esse valor fornece uma estimativa análoga a um procedimento de padronização indireta de taxas de mortalidade, em que se calculam os óbitos esperados caso a população livre apresentasse a estrutura das taxas de mortalidade escrava. Dessa forma, a diferença entre taxas médias estimadas de mortalidade geral atribuídas a escravos e livres pode ser decomposta em:

$\bar{M}_{E}-\bar{M}_{L}=\left[\bar{M}_{E}-\tilde{M}_{L}\right]+\left[\tilde{M}_{L}-\tilde{M}_{L}\right]$

0 primeiro termo entre colchetes do lado direito da equação 3 mede o chamado efeito das características específicas de escravos e livres sobre a probabilidade de morte no hospital. Também é conhecido como efeito composição, ou efeito explicado da decomposição. Suponha-se, por exemplo, que pacientes casados apresentem menor probabilidade de morte do que não casados. Se a população escrava apresentar maior proporção de não casados do que a livre, o efeito das características terá sinal positivo e contribuirá para um aumento do diferencial entre livres e escravos.

0 segundo termo contabiliza o efeito dos coeficientes da regressão nas diferenças de mortalidade entre escravos e livres. Esse efeito é comumente tratado como uma medida de discriminação na Economia do Trabalho: trata-se de um efeito "não explicado" pelas características da população, podendo ser comparado a um efeito da estrutura das taxas específicas de mortalidade, como em Kitagawa (1955). Suponha-se, por exemplo, que mulheres escravas estejam mais expostas a complicações no parto. Uma mulher livre com as mesmas características terá menor probabilidade de morte devido a um efeito dos coeficientes de sinal positivo, contribuindo para um aumento do diferencial entre livres e escravos.

Pode ser interessante analisar os efeitos específicos de cada variável sobre o diferencial de mortalidade, como nos dois exemplos anteriores, em que usamos efeitos específicos 
das variáveis estado conjugal e sexo para explicar o conceito por trás da decomposição. No entanto, uma limitação da decomposição de Oaxaca-Blinder original é o chamado problema de identificação dos grupos de referência. Isso significa que o resultado da decomposição varia conforme se escolhem diferentes grupos de referência da regressão. De outro modo, a decomposição é sensível de acordo com a escolha do padrão de referência. Esse problema foi discutido em Scorzafave e Pazello (2007), sendo aplicada a solução proposta por Yun (2005b), que consiste em restringir os coeficientes das variáveis binárias a categorias individuais de soma zero, ou seja, utilizar um conjunto de "pesos" de modo que os resultados da decomposição sejam invariantes à escolha do padrão de referência. A contribuição relativa de cada variável para o diferencial de mortalidade, em termos de efeitos das características e efeitos dos coeficientes, é dada por:

$\bar{M}_{E}-\bar{M}_{L}=\sum_{i=1}^{K} W_{\Delta X}^{K}\left[\bar{M}_{E}-\tilde{M}_{L}\right]+\sum_{i=1}^{K} W_{\Delta \beta}^{K}\left[\tilde{M}_{L}-\bar{M}_{L}\right]$

Em que os pesos $W_{\Delta X}^{K}$ e $W_{\Delta \beta}^{K}$ indicam a contribuição relativa da variável $k$ em termos de efeitos das características e dos coeficientes. Essa decomposição é livre do problema de grupos de referência. 0 cálculo dos pesos pode ser realizado a partir das informações dos coeficientes da regressão e dos valores médios das variáveis do modelo, como proposto por Yun (2004, p. 277-278), e a operacionalização desses cálculos se deu a partir da rotina escrita por Jann (2008).

Dos 4.208 registros das matrículas entre outubro de 1876 e dezembro de 1885, foram utilizados na análise 3.822 (1.262 escravos e 2.560 livres) que excluem informações incompletas. Descartar os casos para os quais não há informações em pelo menos uma das variáveis contempladas na análise implica uma desigualdade entre a diferença das probabilidades de morte e a diferença entre as proporções de óbito observadas, dado que se tratará de uma aproximação. ${ }^{5}$

\section{Resultado e discussão}

A taxa de mortalidade hospitalar era elevada na Santa Casa de Misericórdia de Campinas e esse perfil pode ser considerado uma regra para os hospitais do século XIX. Outras Misericórdias apresentavam desempenho semelhante, como podemos observar a partir dos Relatórios dos Presidentes de Província. A título de comparação, a Santa Casa de Santos registrou, para os anos de 1879/1880, uma taxa de mortalidade de 15,2\% das internações e, para o ano seguinte, de cerca de 12\% (SÃO PAULO, 1881, Livro 1025, p. 250).

A partir dos dados de Claudia Tomaschewski (2007), observamos que as taxas brutas de mortalidade hospitalar de Campinas se assemelhavam à da Misericórdia de Porto Alegre e foram superiores, em todos os anos comparados, a Pelotas, ultrapassando inclusive

\footnotetext{
5 Esse procedimento poderia ser problemático em pequenas amostras. Alternativamente, é possível utilizar métodos de imputação para estimar a mortalidade hospitalar a partir dos casos desconhecidos. No entanto, essa escolha metodológica também introduz vieses.
} 
em seu primeiro ano de funcionamento $20 \%$ das entradas totais. A Santa Casa do Rio de Janeiro, com movimentação de mais de 15.000 enfermos por ano, manteve para o período $1877 / 1878$ uma taxa bruta de mortalidade hospitalar de $15,8 \%$, registrando $15,4 \%$, no ano seguinte, e 15\%, em 1882 (BRASIL, 1878, 1879, 1882). Em suma, hospitais das Santas Casas de vários tamanhos e cidades apresentavam taxas semelhantes com populações e em condições diversas, o que requer um estudo comparativo mais aprofundado que não se concentre somente no caso de uma localidade.

Uma visão mais geral do contingente em estudo encontra-se na Tabela 1, que apresenta as características dos enfermos da Santa Casa de Misericórdia de Campinas, segundo sexo, estado conjugal e local de residência. As características de escravos e brasileiros livres são próximas em proporções no que se refere à composição por sexo e estado conjugal. Os homens representavam $66 \%$ do total da entrada de escravos, mesma proporção observada para brasileiros livres. Os cativos que se declararam casados correspondiam a $25 \%$, ou seja, proporção semelhante à dos nacionais. As diferenças revelaram-se mais significativas quando consideramos o local de residência: escravos residentes em Campinas eram $67 \%$ relativamente a $88 \%$ de nacionais livres. Isso provavelmente deve-se à limitação da própria construção da variável: acreditamos que, ao anotar "Campinas" como local de residência, o escrivão referia-se ao quadro urbano; caso contrário o nome da fazenda aparece como local de residência. Assim, há evidência da predominância numérica masculina, dos solteiros e urbana na população em questão.

TABELA 1

Características dos enfermos da Santa Casa de Misericórdia de Campinas em termos de proporção relativa - 1876-1885

\begin{tabular}{|c|c|c|c|c|}
\hline Variáveis & Escravos & Livres & $\begin{array}{c}\text { Brasileiros } \\
\text { livres }\end{array}$ & $\begin{array}{c}\text { Estrangeiros } \\
\text { livres }\end{array}$ \\
\hline \multicolumn{5}{|l|}{ Variável dependente } \\
\hline Morte hospitalar & 0,185 & 0,150 & 0,196 & 0,106 \\
\hline \multicolumn{5}{|l|}{ Sexo } \\
\hline Homens & 0,663 & 0,784 & 0,663 & 0,898 \\
\hline Mulheres & 0,337 & 0,216 & 0,337 & 0,102 \\
\hline \multicolumn{5}{|l|}{ Estado conjugal } \\
\hline Casado & 0,253 & 0,282 & 0,252 & 0,311 \\
\hline Não casado & 0,747 & 0,718 & 0,748 & 0,689 \\
\hline \multicolumn{5}{|l|}{ Local de residência } \\
\hline Campinas & 0,666 & 0,883 & 0,859 & 0,907 \\
\hline Outras localidades & 0,334 & 0,117 & 0,141 & 0,093 \\
\hline Sobreviventes & 1.160 & 2.391 & 1.095 & 1.296 \\
\hline Mortos & 248 & 409 & 261 & 148 \\
\hline Total de observações & 1.408 & 2.800 & 1.356 & 1.444 \\
\hline
\end{tabular}

Fonte: Matrículas de Enfermos do Hospital de Misericórdia de Campinas (1876-1885).

A distribuição etária por condição social traz fortes evidências de viés significativo para indivíduos do sexo masculino, em especial para estrangeiros. Na Santa Casa, a maioria dos 
estrangeiros livres atendidos era de portugueses, que representavam cerca de $30 \%$ do total dos estrangeiros (407 homens e 22 mulheres), seguidos por italianos (29\%), franceses (9\%), alemães (9\%) e africanos livres (8\%).0 Gráfico 1 mostra a distribuição etária por grupos quinquenais, em que fica evidente o perfil diferenciado da população estrangeira e relativamente semelhante da população escrava com os brasileiros livres.

\section{GRÁFICO 1}

Distribuição dos enfermos da Santa Casa de Misericórdia de Campinas, por grupos de idade, segundo condição social - 1876-1885

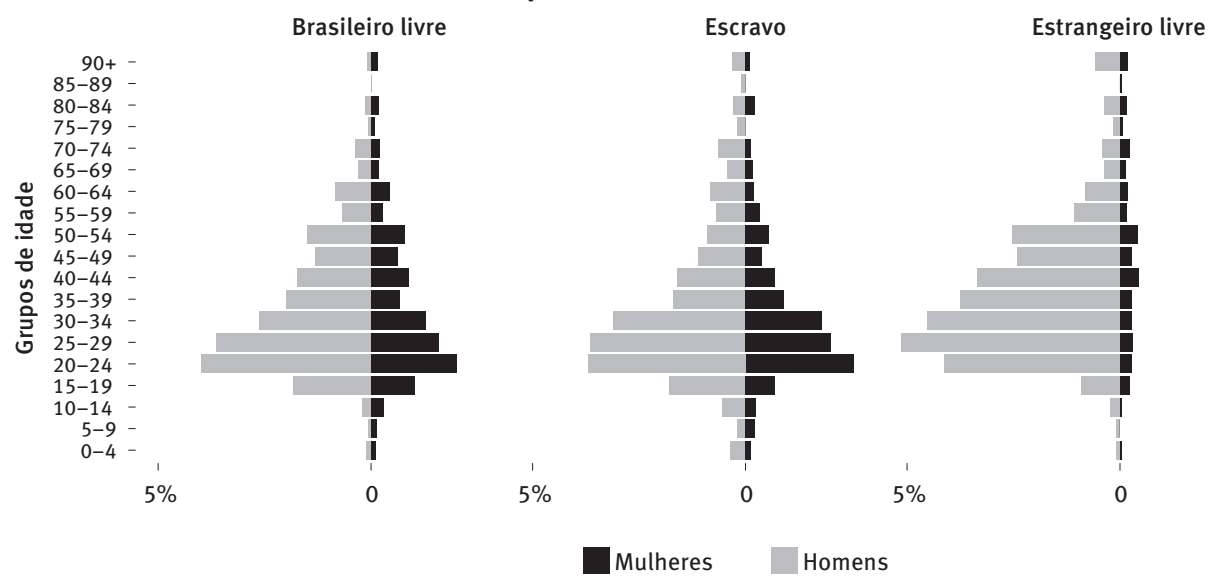

Fonte: Matrículas de Enfermos do Hospital de Misericórdia de Campinas (1876-1885).

Os resultados do Censo de 1872 e das Matrículas são semelhantes nas primeiras faixas de idade, mas o viés de indivíduos do sexo masculino na lista de pacientes do hospital é intenso. As razões de sexos mostram uma sobrerrepresentação dos homens em idade ativa no hospital, sendo um pouco menor entre os pacientes escravos (Gráfico 2). Essa distorção é provocada por estrangeiros, que têm composição acentuadamente masculina. Além disso, nota-se uma distorção significativa nos resultados para as últimas faixas de idade, o que sugere um problema de exagero na declaração de idade nesses dados. Essa representação de estrangeiros, sobretudo de portugueses, alemães, italianos e africanos livres, é significativa mesmo considerando a população total. O Censo de 1872 mostra que $78 \%$ da população estrangeira livre da cidade era composta por homens. Campinas abrigava 13,11\% do total da população estrangeira do Estado de São Paulo.

A análise das taxas específicas de mortalidade hospitalar nos conduz a outro ponto importante: o diferencial com relação ao sexo. Usualmente, as diferenças de mortalidade entre homens e mulheres são apresentadas em termos de razão de sobremortalidade masculina. No entanto, para a Santa Casa de Misericórdia, mulheres pouco foram atendidas e morreram na maior parte dos casos, de forma que a relação inversa melhor ilustra o resultado principal. Dessa forma, procedemos com o cálculo das taxas específicas de mortalidade hospitalar, relacionando a população feminina com a masculina. 0 Gráfico 3 revela que, para as faixas de idade reprodutiva, as mulheres morreram quase duas vezes mais do que os homens. 


\section{GRÁFICO 2}

Razão de sexos da população total e escrava de Campinas (1874) e da Santa Casa de Misericórdia, por grupos de idade - 1876-1885

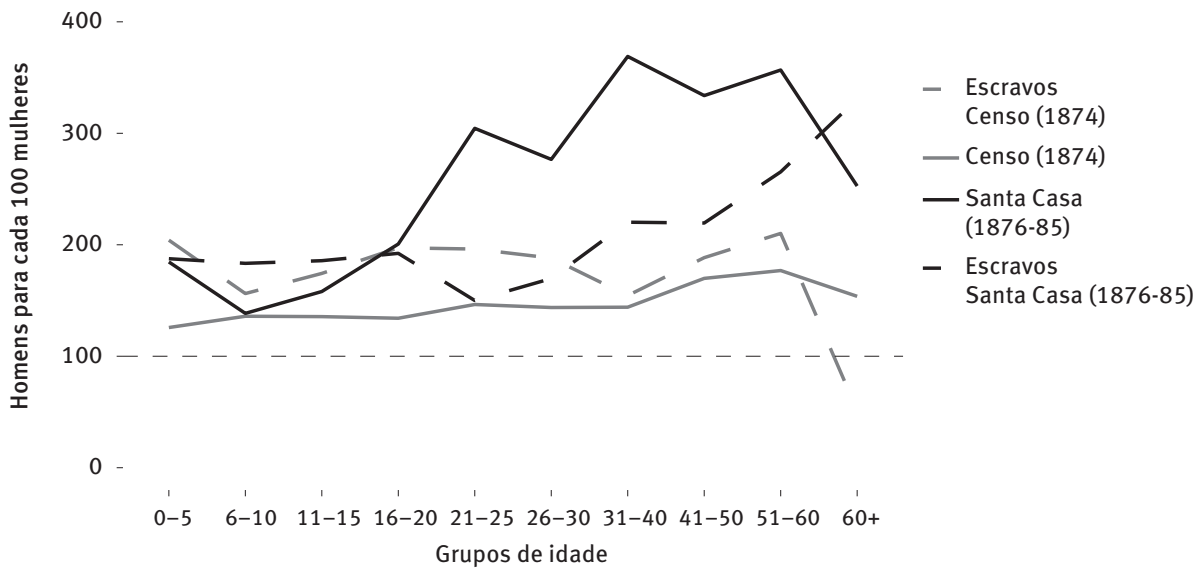

Fonte: Dados do Censo de 1872 extraídos de Bassanezi (1998) e Matrículas de Enfermos do Hospital de Misericórdia de Campinas (1876-1885).

Daniel Oliveira (2012) mostra um padrão semelhante na Santa Casa de Porto Alegre. Geralmente, as mulheres chegavam aos hospitais em situação mais grave, depois de esgotados os tratamentos tradicionais realizados por curandeiros ou parteiras, no caso de complicações no parto. Na Santa Casa de Campinas, observamos alguns casos de inflamações no útero e doenças puerperais que, de um modo geral, não são representativos, mas acumulam-se a todas as outras doenças infectocontagiosas que tiveram grande participação no número de óbitos. A mortalidade feminina não se comportou da mesma forma nas populações escrava e livre, cabendo investigar os diferenciais de mortalidade quanto à condição social.

\section{GRÁFICO 3}

Sobremortalidade das mulheres na Santa Casa de Misericórdia de Campinas, por grupos de idade - 1876-1885

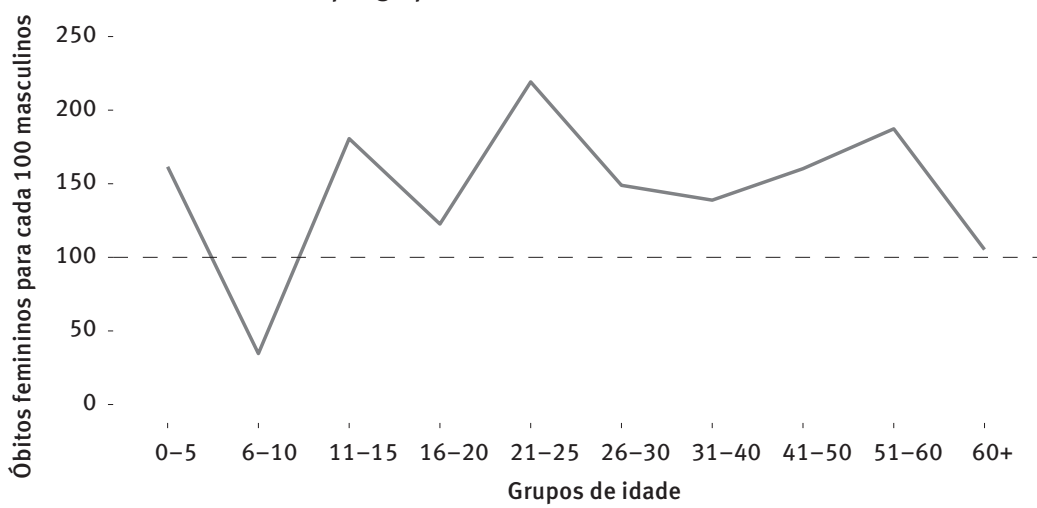

Fonte: Matrículas de Enfermos da Santa Casa de Misericórdia de Campinas (1876-1885).

A diferença entre a mortalidade de escravos e a de livres estimada foi de três pontos percentuais. Em 1881, quando a mortalidade escrava chegou a $24,7 \%$ e a dos livres 
alcançou $12 \%$, esses resultados foram interpretados como "ótimos” para os escravizados e elevados para a população livre. Segundo o médico Guilherme da Silva, o resultado quanto à classe escrava é relativamente ótimo, porque alguns fazendeiros consideravam a Santa Casa "um verdadeiro necrotério por onde devem passar os escravos". Por sua vez, os doentes livres só buscavam tratamento quando esgotadas todas as alternativas muitas vezes recomendadas por leigos, o chamado batismo terapêutico. Chegavam em estado grave ou avançado das doenças, tornando a terapêutica impotente perante eles (RELATÓRIO DOS PROVEDORES, 1881, p. 11).

TABELA 2

Decomposição do diferencial de mortalidade hospitalar entre escravos e livres na Santa Casa de Misericórdia de Campinas - 1876-1885

\begin{tabular}{|c|c|c|c|c|c|}
\hline Variáveis & $\begin{array}{c}\text { Efeito das } \\
\text { características }\end{array}$ & $\begin{array}{c}\text { Diferença } \\
(\%)\end{array}$ & $\begin{array}{c}\text { Efeito dos } \\
\text { coeficientes }\end{array}$ & $\begin{array}{c}\text { Diferença } \\
(\%)\end{array}$ & $\begin{array}{c}\text { Efeito total } \\
(\%)\end{array}$ \\
\hline Efeito agregado & $-0,022^{\star \star \star}$ & $-72,2$ & $0,052^{\star \star \star}$ & 172,2 & 100,0 \\
\hline \multicolumn{6}{|l|}{ Efeitos específicos } \\
\hline Idade & 0,001 & $-2,9$ & $-0,027$ & $-91,4$ & $-88,5$ \\
\hline Sexo & 0,001 & 2,5 & $0,033^{\star \star \star}$ & 110,9 & 113,3 \\
\hline Estado conjugal & $0,001^{\star \star}$ & 4,3 & 0,004 & 13,6 & 17,9 \\
\hline Local de residência & $-0,012^{\star \star}$ & $-39,2$ & $0,037^{\star \star}$ & 122,1 & 82,9 \\
\hline Permanência hospitalar & $-0,007^{\star \star \star}$ & $-21,9$ & 0,000 & 1,6 & $-20,3$ \\
\hline Mês de admissão & $-0,003$ & $-8,9$ & 0,000 & 1,5 & $-7,4$ \\
\hline Ano de admissão & $-0,004^{\star \star \star}$ & $-11,9$ & $-0,004$ & $-12,6$ & $-24,5$ \\
\hline Intercepto & - & - & $-0,008$ & 26,5 & 26,5 \\
\hline $\begin{array}{l}\text { Escravos }(\mathrm{N}=1.262) \\
\text { Livres: brasileiros }+ \\
\text { estrangeiros }(\mathrm{N}=2.560) \\
\text { Diferencial de mortalidade }\end{array}$ & & & $\begin{array}{l}0,176 \\
0,146 \\
0,030^{\star \star \star}\end{array}$ & & \\
\hline
\end{tabular}

Fonte: Matrículas de Enfermos do Hospital de Misericórdia de Campinas (1876-1885).

Nota: ${ }^{\star \star *},{ }^{\star *}$, * denotam significância de $1 \%, 5 \%$ e $10 \%$, respectivamente. Decomposição de Oaxaca-Blinder operacionalizada por meio da rotina criada por Jann (2008).

0 efeito das características apresentou sinal negativo, respondendo por $72,2 \%$ do diferencial de mortalidade. Isso significa que escravos acumularam características demográficas com efeito atenuante das diferenças de mortalidade na ordem de 2,2 pontos percentuais. No entanto, o efeito agregado dos coeficientes foi no sentido de agravar as diferenças entre escravos e livres e representou $172,2 \%$ da mortalidade diferencial, correspondendo a 5,2 pontos percentuais de diferença. Em outras palavras, apesar de a população escrava apresentar composição favorável a uma menor mortalidade em média, a maior probabilidade de morte decorrente da condição de escravo é responsável pela mortalidade diferencial.

O efeito dos coeficientes é muitas vezes entendido como uma parte "não explicada" das diferenças, ou como um termo de “discriminação". Quando analisamos os efeitos das variáveis individualmente, é possível verificar que $44 \%$ desse efeito não explicado decorre da variável sexo. Ou seja, há um efeito agravante da mortalidade diferencial de 3,3 pontos percentuais relacionado ao sexo, que é responsável pelas diferenças de mortalidade entre escravos e livres. Quais hipóteses poderiam ser levantadas a esse respeito? No geral, como a mortalidade materna foi mais elevada, essa característica da população atuaria no sentido 
de reduzir a mortalidade escrava e a diferença com relação aos livres. Entretanto, isso não se verificou na prática. Homens livres apresentaram de fato probabilidades de morte menores do que as mulheres livres, mas isso não ocorreu para a população escrava, em que homens e mulheres apresentaram níveis de mortalidade semelhantes. A significância estatística das variáveis de ano de admissão sugere ainda que existem efeitos de período.

Havia incentivos econômicos em um bom tratamento dos escravos. Segundo Pritchett e Yun (2009, p. 22), a partir do momento que o escravo era entendido como um investimento pelo seu senhor e a diária nos leitos do hospital tinha um custo, o senhor de escravos provia o tratamento quando seu benefício marginal - isto é, o diferencial entre o preço do escravo saudável e seu preço depreciado devido à enfermidade - excedia os custos de hospitalização. ${ }^{6}$ Lander e Pritchett (2009) apontam que o cuidado dado a escravos poderia estar associado ao seu preço de mercado. Velhos e crianças raramente recebiam atendimento no hospital de Touro, indicando que só escravos mais valiosos recebiam tratamento.

É notável que os escravos atendidos pela Santa Casa de Campinas acumulavam características consideradas geralmente valorizadas, visto que foram majoritariamente homens em idade ativa. ${ }^{7}$ Dessa forma, poderia não haver incentivos econômicos na internação hospitalar de escravos menos relevantes à economia dos proprietários, sobretudo mulheres e indivíduos fora da idade ativa. Além disso, um fator responsável pela maior mortalidade feminina comumente identificado pela bibliografia refere-se aos casos cirúrgicos de mortalidade materna. Para essas situações, os escravos contavam com um sistema próprio de assistência que inclui curandeiros, sangradores e parteiras - que muitas vezes também era utilizado por brancos (PÔRTO, 2006, p. 1023).

Outra variável exerce influência significativa para a diferença "não explicada" entre escravos e livres: o estado conjugal. No geral, há tendência de os indivíduos casados apresentarem melhor saúde do que os não casados. No entanto, esse efeito foi ainda maior para a população escravizada. Escravos casados tiveram mortalidade hospitalar reduzida em maior intensidade do que os nacionais e estrangeiros nessa mesma condição conjugal. Como escravos têm maior representação de não casados do que os livres, o efeito das características dessa variável contribuiu para um agravamento do diferencial. Além do mais, o efeito dos coeficientes aponta para o mesmo sentido, de forma que essa variável contribui para $11 \%$ das diferenças totais da mortalidade entre escravos e livres: caso as duas populações apresentassem mesma proporção de não casados, $11 \%$ da mortalidade diferencial desapareceria. Uma análise mais cuidadosa sobre os indivíduos casados pode revelar que, inseridos em laços de solidariedade e num contexto de constituição de família, eles apresentavam realmente maiores chances de resistir à morte devido ao efeito de um “cuidador alternativo" ao hospital.

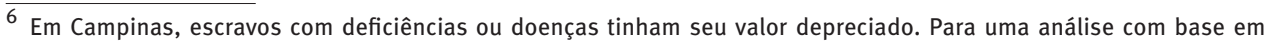
inventários, ver Martins (2004).

7 o preço do escravo era influenciado por suas características demográficas, sexo e idade, valendo para a generalidade dos casos os maiores preços para homens em idade ativa. Para uma análise sobre o preço de escravos conforme suas características, ver Marcondes e Motta (2001).
} 
Uma primeira aproximação dos dados de moléstias revela que a doença que mais causou óbitos na Santa Casa foi sem dúvida a tuberculose, com uma mortalidade de $49 \%$ dos casos (100 óbitos para 202 entradas com esse diagnóstico). Em segundo lugar, vieram algumas doenças cardiovasculares como lesões cardíacas e insuficiências mitral e aórtica, que foram responsáveis por uma mortalidade de $44 \%$ dos casos (63 óbitos para 143 entradas). Diarreias e enterites provocaram vítimas fatais em $33 \%$ dos casos (62 óbitos para 187 entradas) e doenças respiratórias como pneumonia e broncopneumonia, com óbitos em $31 \%$ dos diagnósticos. Em suma, predominavam as doenças associadas às condições de vida e de trabalho.

Dentro da população livre campineira existiram diferenças importantes na probabilidade de morte. Para todos os anos, brasileiros livres apresentaram taxas de mortalidade maiores do que estrangeiros no hospital da Santa Casa.

As estimativas da decomposição da mortalidade diferencial apontam para uma diferença média entre brasileiros e estrangeiros livres de 8,9 pontos percentuais: maior do que a diferença entre escravos e livres de maneira agregada. A Tabela 3 apresenta a decomposição do diferencial em termos agregados e específicos para cada variável explicativa do modelo. Sinais negativos das estimativas têm efeito atenuante, enquanto sinais positivos têm efeito agravante no diferencial entre brasileiros e estrangeiros. Nesse caso, tanto o efeito das características quanto o efeito dos coeficientes vão no sentido de agravar a mortalidade diferencial em favor dos estrangeiros. 0 efeito agregado das características representou $26,7 \%$ do diferencial total, isto é, 2,4 pontos percentuais. Isso se deve especialmente a diferenças na representação feminina nas duas populações.

TABELA 3

Decomposição do diferencial de mortalidade hospitalar entre brasileiros e estrangeiros na Santa Casa de Misericórdia de Campinas - 1876-1885

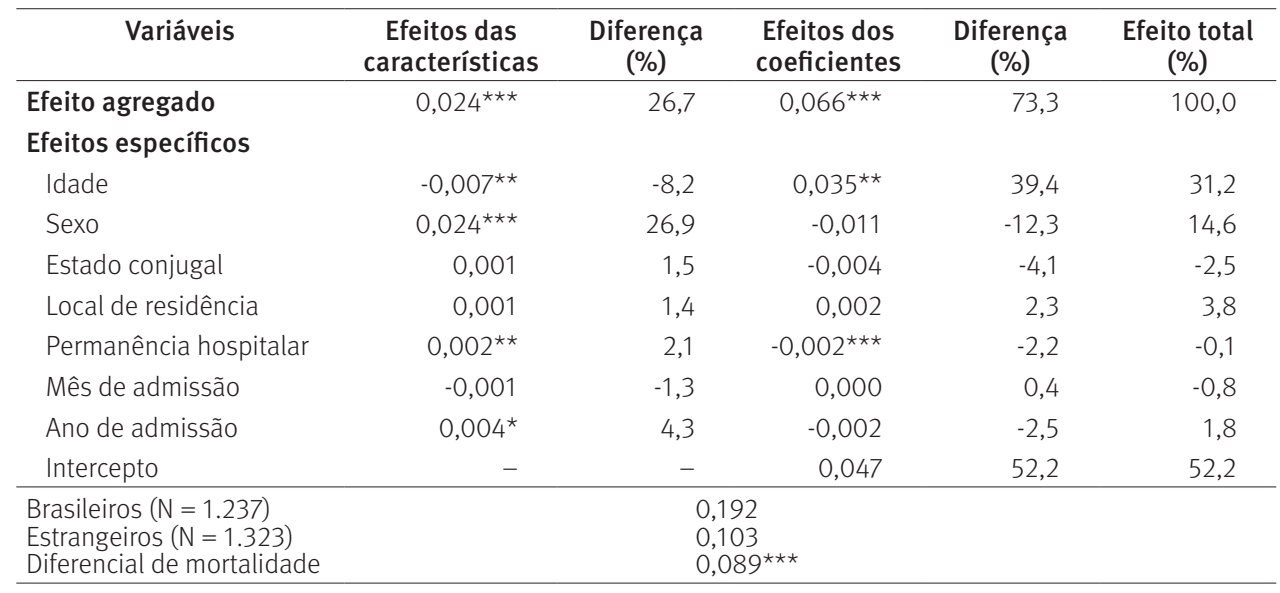

Fonte: Matrículas de Enfermos do Hospital de Misericórdia de Campinas (1876-1885).

Nota: ${ }^{* \star},{ }^{* *}$, * denotam significância de 1\%, 5\% e 10\%, respectivamente. Decomposição de Oaxaca-Blinder operacionalizada por meio da rotina criada por Jann (2008).

Como explorado, o perfil dos enfermos da Santa Casa é enviesado para o sexo masculino, especialmente entre os estrangeiros. Muito disso deve-se à própria composição da 
população estrangeira do município, que, sendo predominantemente homens de idade ativa, era mão de obra em potencial. Mulheres morreram mais no Hospital de Misericórdia, motivo pelo qual a menor representação feminina entre os estrangeiros lhes conferiu um "bônus" de 2,4 pontos percentuais de diferença sobre a mortalidade de brasileiros via efeito das características. Um fator que poderia contribuir para essa sub-representação feminina e sobremortalidade era a visão comum à época de que os hospitais eram lugares masculinos, inadequados para mulheres e moças. Essa impressão é apontada por lan Read (2012, p. 134) estudando a Santa Casa de Santos, diante de uma representação feminina de apenas $13 \%$ entre os enfermos livres. Apesar das distinções e do perfil de Santos como cidade portuária, recebendo um grande número de marinheiros, as mulheres evitavam os cuidados dos hospitais e se apresentavam em casos graves.

Outras razões foram identificadas por Renilda Barreto (2001) na Santa Casa da Bahia. A autora aponta que as enfermarias inspiravam desconfiança, além de haver certa preferência pelo diagnóstico e cura das enfermidades realizados por outras mulheres, na privacidade do lar. Para a autora, a Santa Casa foi procurada especialmente pelas mulheres pobres, presas, escravas e desvalidas, sem condições de receber os cuidados na esfera privada (BARRETO, 2001, p. 140-141). Pesaram contra elas os casos de morte materna, que aumentam as chances de mortalidade hospitalar em casos graves e impactam nos diferenciais por sexo.

Em sentido contrário, o efeito das características atribuído à estrutura etária das populações favorece uma diminuição no diferencial em favor dos brasileiros. Esse efeito não é suficiente para compensar os efeitos da variável sexo, mas reduzem a mortalidade diferencial em 0,7 ponto percentual. Como uma idade mais avançada tem efeito positivo sobre a mortalidade e diante de uma proporção de adultos e idosos maior entre os estrangeiros, esse efeito contribuiria para uma diminuição do diferencial.

No entanto, o efeito-idade sobre a mortalidade não é o mesmo nesses dois grupos. A parte "não explicada" pelas características correspondeu a 73,3\% do diferencial de mortalidade, em grande parte por diferenças na estrutura etária. Enquanto o perfil etário foi associado a uma mortalidade mais elevada dos brasileiros, o efeito entre os estrangeiros foi bem próximo de zero. Disso resulta que, se todas as características que observamos de brasileiros e estrangeiros fossem iguais em média, $73,3 \%$ do diferencial de mortalidade permaneceria. Quais os fatores não observados que poderiam levar a uma mortalidade de brasileiros maior do que a de estrangeiros?

0 relatório do ano compromissal de 1882-1883 trouxe evidências de que o hospital era concorrido de preferência por estrangeiros que vinham prestar seu braço ao trabalho e eram acometidos pelo cansaço e pelas doenças (RELATÓRIO DOS PROVEDORES, 1883, p. 11). Na mentalidade da população nacional, o hábito de procurar a Misericórdia (e outros hospitais) seria enraizado mais lentamente. Ainda que tais considerações para a mortalidade diferencial permitam explicar uma parcela das diferenças entre homens e mulheres, escravos e livres, nacionais e estrangeiros, parte desse diferencial permanece não explicada e requer maior investigação acerca das hipóteses levantadas, da influência 
de outras variáveis como nível de renda e escolaridade, bem como do padrão de morbidade das principais doenças trazidas por outras fontes, que podem fornecer elementos para estudos sobre o acesso à saúde e comportamento da morbimortalidade durante 0 final do século XIX.

\section{Considerações finais}

A análise da mortalidade diferencial mostrou que os escravos, apesar de reunirem características que diminuíram as chances de morte hospitalar e atenuantes do diferencial de mortalidade, morreram em maior quantidade dos casos. Havia incentivos econômicos para o tratamento de escravos homens em idade ativa e no atendimento o mais rápido possível dessa categoria, visto que eram considerados mão de obra valiosa. Ainda assim, argumentamos que, devido a uma condição de vida e trabalho mais precária imposta pelo cativeiro, a mortalidade escrava foi ligeiramente superior à dos livres. Mesmo em condições de tratamento aparentemente mais “igualitárias", os diferenciais de mortalidade entre escravizados e livres persistem. Uma pesquisa mais aprofundada a respeito dos sistemas de assistência aos escravos disponíveis para Campinas ainda merece atenção.

Dentro da população livre também existiram consideráveis diferenças entre nacionais e estrangeiros. Para todos os anos analisados, os brasileiros livres apresentaram taxas de mortalidade maiores do que estrangeiros na Santa Casa. Uma parcela dessa diferença refletiu-se nas características da distribuição etária das duas populações, sub-representação feminina e outros fatores pouco explicados. Uma hipótese que ajudou a compreender essa mortalidade diferencial estabeleceu-se na procura tardia dos nacionais por auxílio do hospital, muito devido ao estigma de ser considerado um lugar com altíssimas taxas de mortalidade. Tendo isso em vista, nacionais procuravam o hospital com enfermidades muitas vezes em estado grave, avançado ou tendo feito uso de terapias alternativas. Ademais, a sub-representação feminina teve impacto agravante na mortalidade diferencial entre brasileiros e estrangeiros. Parte disso devido à própria estrutura populacional desse segmento e parte revelada por meio da visão comum à época de que os hospitais eram lugares masculinos, fazendo com que raramente mulheres chegassem ao hospital.

Por fim, essa exploração abre algumas possibilidades para pesquisas voltadas ao acesso à saúde e ao estudo da dinâmica da morbimortalidade da população escrava, nacional e estrangeira, especialmente dos que não podiam pagar serviços privados de assistência.

\section{Referências}

BAHIA. Relatório com que ao illm. e exm. snr. desembargador Henrique Pereira de Lucena, passou a administração da província em 5 de fevereiro de 1877 o exm snr. conselheiro Luiz Antonio da Silva Nunes. Bahia: Typographia do Jornal da Bahia, 1877.

BARRETO, R. Corpo de mulher: a trajetória do desconhecido na Bahia do século XIX. História: questões e debates, Curitiba, n. 34, p. 127-156, 2001. 
BRASIL. Relatório apresentado à Assembleia Geral Legislativa na 1a sessão da 17a legislatura pelo Ministro e Secretário de Estado dos Negócios do Império, Carlos Leoncio de Carvalho. Rio de Janeiro, 1878.

BRASIL. Relatório apresentado à Assembleia Geral Legislativa na 2a sessão da 17a legislatura pelo Ministro e Secretário de Estado dos Negócios do Império, Carlos Leoncio de Carvalho. Rio de Janeiro, 1879.

BRASIL. Relatório apresentado à Assembleia Geral Legislativa na 1a sessão da 18a legislatura pelo Ministro e Secretário de Estado Interino dos Negócios do Império, Manoel Pinto de Souza Dantas. Rio de Janeiro, 1882.

BASSANEZI, M. S. C. B. São Paulo do passado: dados demográficos. Campinas, 1998. v. 1-5.

BLINDER, A. S. Wage discrimination: reduced form and structural estimates. The Journal of Human Resources, Madison, v. 8, n. 4, p. 436-455, 1973.

CANUDAS ROMO, V. Decomposition methods in demography. Tese (Doutorado) - Faculty of Spatial Sciences, University of Groningen, Groningen, 2003.

CHERRY, S. The hospitals and population growth: the voluntary general hospitals, mortality and local populations in the English provinces in the eighteenth and nineteenthcenturies - Part 2. Population Studies, v. 34, n. 2, p. 251-265, 1980.

COSTA, I. Vila Rica: população (1718-1826).São Paulo: IPE-USP, 1979.

DAS GUPTA, P. A general method of decomposing a difference between two rates into several components. Demography, v. 15, n. 1, p. 99-112, 1978.

EISENBERG, P. Homens esquecidos: escravos e trabalhadores livres no Brasil - séculos XVIII e XIX. Campinas: Unicamp, 1989.

FERRIE, J. P. The rich and the dead: socioeconomic status and mortality in the united states, 1850-1860. In: COSTA, D. (Ed.). Health and labor force participation over the life cycle: evidence from the past. Chicago: University of Chicago Press, 2003. p. 11-50.

GREEN, T. L.; HAMILTON, T. G. Beyond black and white: color and mortality in post-reconstruction era North Carolina. Explorations in Economic History, v. 50, n. 1, p. 148-159, 2013.

HORIUCHI, S. Epidemiological transitions in human history. Health and mortality: issues of global concern. New York, United Nations, 1999. p. 54-71.

JANN, B. A Stata implementation of the Blinder-Oaxaca decomposition. Stata Journal, v. 8, n. 4, p. 453-479, 2008.

KARASCH, M. A vida dos escravos no Rio de Janeiro, 1808-1850. São Paulo: Companhia das Letras, 2000.

KITAGAWA, E. M. Components of a difference between two rates. Journal of the American Statistical Association, v. 50, n. 272, p. 1168-1194, 1955.

LANDER, K.; PRITCHETT, J. When to care: the economic rationale of slavery health care provision. Social Science History, v. 33, n. 2, p. 155-182, 2009.

LAPA, J. R. do A. A cidade: os cantos e os antros - Campinas, 1850-1900. São Paulo: Edusp, 1996.

MARCONDES, R. L.; MOTTA, J. F. Duas fontes documentais para o estudo dos preços dos escravos no Vale do Paraíba paulista. Revista Brasileira de História, v. 21, n. 42, p. 495-514, 2001.

MARTINS, V. Livres, escravos e doenças: Campinas século XIX. In: ENCONTRO REGIONAL DE HISTÓRIA, 17. Anais...Campinas: Unicamp, 2004. 
MATRÍCULAS de Enfermos do Hospital da Santa Casa de Misericórdia de Campinas. Arquivo do Centro de Memória Unicamp, 1876-1885.

MESGRAVIS, L. A Santa Casa de Misericórdia de São Paulo (1599?-1884): contribuição ao estudo da assistência social no Brasil. São Paulo: Conselho Estadual de Cultura, 1977.

OAXACA, R. Male-female wage differentials in urban labor markets. International Economic Review, v. 14, n. 3, p. 693-709, 1973.

OLIVEIRA, D. Morte e vida feminina: mulheres pobres e medicina da mulher na Santa Casa de Misericórdia de Porto Alegre (1800-1900). Dissertação (Mestrado em História) - Instituto de Filosofia e Ciências Humanas, Universidade Federal do Rio Grande do Sul, Porto Alegre, 2012.

OMRAM, A. R. The epidemiologic transition: a theory of the epidemiology of population change. Milbank Memorial Fund Quarterly, v. 49, n. 1, p. 509-538, 1971.

PORTO ALEGRE. Falla com que o exm. sr. dr. João Chaves Campello abrio a segunda sessão da 17a legislatura no dia 12 de março de 1878. Porto Alegre: Typographia do Mercantil, 1878.

PÔRTO, Â. O sistema de saúde do escravo no Brasil do século XIX: doenças, instituições e práticas terapêuticas. História, Ciências, Saúde-Manguinhos, v. 13, n. 4, 2006.

PRITCHETT, J.; YUN, M.-S. The in-hospital mortality rates of slaves and freemen: evidence from Touro Infirmary, New Orleans, Louisiana, 1855-1860. Explorations in Economic History, v. 46, n. 2, p. 241-252, 2009.

READ, I. The hierarchies of slavery in Santos, Brazil, 1822-1888. Stanford: Stanford University Press, 2012.

RELATÓRIO apresentado à Irmandade de Misericórdia de Campinas em sua reunião geral de 26 de junho de 1881 pelo seu provedor Joaquim José Vieira. Campinas: Typographia da Gazeta de Campinas, 1881.

RELATÓRIO apresentado à Irmandade de Misericórdia de Campinas pelo respectivo provedor Joaquim José Vieira. São Paulo: Typographia da Província, 1877.

RELATÓRIO apresentado à Irmandade de Misericórdia de Campinas pelos respectivos provedores cônego Joaquim José Vieira efetivo e Dr. Valentim José da Silveira Lopes interino no ano compromissal de 1882 a 1883. São Paulo: Typographia a Vapor da Gazeta, 1883.

ROCHA, L. A. Caridade e poder: a Irmandade da Santa Casa de Misericórdia de Campinas (18711889). Dissertação (Mestrado em História Econômica) - Instituto de Economia, Universidade Estadual de Campinas (IE/Unicamp), Campinas, 2005.

RUSSELL-WOOD, A. J. Fidalgos e filantropos: a Santa Casa da Misericórdia da Bahia - 1550-1755. Brasília: Editora da Universidade de Brasília, 1981.

SÃO PAULO. Relatório apresentado à Assembléa Legislativa Provincial de S. Paulo pelo presidente da provincia, o exm. sr. dr. Sebastião José Pereira. Santos, 1877.

SÃO PAULO. Relatório apresentado à Assembléa Legislativa Provincial de S. Paulo pelo presidente da provincia, Laurindo Abelardo de Brito, no dia 13 de janeiro de 1881. Santos, 1881.

SCORZAFAVE, L. G.; PAZELLO, E. T. Using normalized equations to solve the indetermination problem in the Oaxaca-Blinder decomposition: an application to the gender wage gap in Brazil. Revista Brasileira de Economia, v. 61, n. 4, p. 535-548, 2007.

SINGER, P.; CAMPOS, O.; OLIVEIRA, E. M. Prevenir e curar: o controle social através dos serviços de saúde. Rio de Janeiro: Forense Universitária, 1981. 
TELAROLLI JUNIOR, R. Assistência sanitária e condições de saúde na zona rural paulista na primeira república. Revista Brasileira de Estudos de População, v. 14, n. 1/2, p. 3-17, 2014.

TOMASCHEWSKI, C. Caridade e filantropia na distribuição da assistência: a Irmandade da Santa Casa de Misericórdia de Pelotas-RS: 1847-1922. Tese (Mestrado em História) - Pontifícia Universidade Católica do Rio Grande do Sul, Porto Alegre, 2007.

WITTER, N. A. Males e epidemias: sofredores, governantes e curadores no sul do Brasil (Rio Grande do Sul, século XIX). Tese (Doutorado em História) - Instituto de Ciências Humanas e Filosofia, Universidade Federal Fluminense, Niterói, 2007.

YUN, M.-S. Decomposing differences in the first moment. Economics Letters, v. 82, n. 2, p. 275 280, 2004.

Hypothesis tests when decomposing differences in the first moment. Journal of Economic and Social Measurement, v. 30, n. 4, p. 295-304, 2005.

. Normalized equation and decomposition analysis: computation and inference. Bonn, Germany, 2005 (IZA Discussion Paper, n. 3652).

\title{
Sobre os autores
}

Matheus Alves Albino é mestrando do Programa de Pós-Graduação em Demografia do Instituto de Filosofia e Ciências Humanas da Universidade Estadual de Campinas (IFCH/Unicamp). Bolsista da Coordenação de Aperfeiçoamento de Pessoal de Nível Superior (Capes) e bacharel em Economia Empresarial e Controladoria pela Faculdade de Economia, Administração e Contabilidade de Ribeirão Preto (FEA-RP/USP).

Renato Leite Marcondes é economista e doutor em Economia pela Universidade de São Paulo. Professor associado do Departamento de Economia da Faculdade de Economia, Administração e Contabilidade de Ribeirão Preto (FEA-RP/USP).

\section{Endereço para correspondência}

\section{Matheus Alves Albino}

Rua dos Tamoios, 439, Vila Miguel Vicente Cury

13081-560 - Campinas-SP, Brasil.

Renato Leite Marcondes

Avenida Bandeirantes, 3900, Edifício da FEA, Vila Monte Alegre

14040-905 - Ribeirão Preto-SP, Brasil

\begin{abstract}
Mortality differentials in a philanthropic hospital: the Santa Casa de Misericórdia of Campinas (1876-1885)

With documentation involving patients' records and reports fromthe administration of Santa Casa de Misericórdia of Campinas (1876-1885), we examined demographic characteristics of patients and analyzed differences in mortality ratesamongslaves, free people, Brazilians, and foreign population atthe philanthropic hospital. Data show patients are predominantly men of working age, mainly involved inlabor available related to agricultural expansion and urban services.
\end{abstract}


Through an analysis of mortality differentials based on the Oaxaca-Blinder Decomposition, we concluded that although the characteristics of slavery and economic incentives in their treatment favored a reduced differential of mortality, slave mortality was higher due to the living conditions imposed by slavery. Among the free population, Brazilians had higher mortality rates than foreigners. Hypotheses raised include abelated demand for help from nationals, differences in age structure, and astrong female underrepresentation among the free population.

Keywords: Hospital mortality. Mortality differentials. Slavery. Santa Casa de Misericórdia.

\section{Resumen}

Diferencias de mortalidad en un hospital filantrópico: Santa Casa de Misericordia de Campinas (1876-1885)

A partir de documentos de las Matrículas de Enfermos e Informes de los Proveedores del Hospital Santa Casa de Misericordia de Campinas, trazamos el perfil de los pacientes y analizamos la mortalidad diferencial entre esclavos y libres, brasileños y extranjeros em ese hospital filantrópico. Los enfermos fueron predominantemente hombres em edad activa, fuertemente relacionados con la mano de obra disponible para la expansión agrícola y de los servicios urbanos. Por medio de una técnica de análisis del diferencial de mortalidad basada en la descomposición de Oaxaca-Blinder, concluimos que a pesar de que las características de los esclavos favorecieron un de mortalidade reducido y que hubo incentivos económicos em su tratamiento, la mortalidad esclava fue superior, posiblemente por las condiciones de vida impuestas por el cautiverio. Entre la población libre, los brasileños presentaron una mortalidad superior a la de población extranjera. Las hipótesis planteadas son: la demanda tardía de ayuda por parte de los nacionales, diferencias en la estrutura etária y la fuerte característica de subrepresentación feminina en la población extranjera.

Palabras clave: Mortalidad hospitalaria. Diferencias de mortalidad. Esclavos. Santa Casa de Misericordia.

Recebido para publicação em 18/11/2018 Aceito para publicação em 19/12/2018 